\title{
Coatings and Aerogels Based on Sol-Gel Processes
}

\author{
Konstantin Siegmann and Martina Hirayama*
}

\begin{abstract}
This article summarizes applications of sol-gel coatings to modify surface properties, and the process is illustrated with several examples. The use of azobenzene-modified silanes leads to a photoswitchable coating displaying light-controlled wettability. Silane coupling reactions are used to coat surfaces with polymers, in order to hinder the ice crystal growth on surfaces. Liquid-like coatings based on poly(ethylene glycol) show anti-adhesive properties to lime. The preparation of aerogels could be improved by selecting proper silanes as coupling agents to the inner surface of the pores. These examples illustrate that sol-gel processes based on silianation reactons are most promising for industrial applications.
\end{abstract}

Keywords: Anti-adhesive · Coating · Interface · Sol-gel · Surface

\section{Introduction}

Surfaces of materials play a crucial role in determining their physical, chemical, mechanical, and optical properties. For this reason, it is essential to be able to tailor surface properties of given materials with appropriate coatings. The aim of such coatings could be to obtain, for example, photo responsive, lime repellent, or insulating surfaces. Sol-gel chemistry is particularly interesting in this context. Sol-gel reactions are normally used in the production of fine powders, monolithic ceramics, glasses,

${ }^{*}$ Correspondence: Prof. Dr. M. Hirayama Institute of Materials and Process Engineering Zurich University of Applied Sciences

Technikumstrasse 9

PO box

$\mathrm{CH}-8401$ Winterthur

$\mathrm{T}+41589347326$

$\mathrm{F}+41589357326$

E-mail: martina.hirayama@zhaw.ch ceramic fibres, inorganic membranes, and aerogels. ${ }^{[1]}$ However, sol-gel reactions can be also used to produce coatings. ${ }^{[2]}$ In the present article, a few possible applications of sol-gel coatings are summarized. The sol-gel reactions are based on alkoxysilanes. In aqueous solution, the precursors undergo hydrolysis and polycondensation reactions to form a colloidal dispersion. Scheme 1 illustrates the condensation process in an acidic medium, but analogous reactions proceed in a basic medium. In the hydrolysis step, the alkoxide is replaced by a hydroxide in a nucleophilic substitution reaction. In the condensation step, the resulting hydroxy-silicon compound reacts with a second silane, forming a dimer, which is held together by a siloxane bridge. Through a sequence of such hydrolysis/ condensation reactions one obtains large colloidal nanocondensates consisting of a siloxane network. Sol-gel coatings can be produced similarly to the wet-chemical procedure discussed above. After cleaning and, if necessary, activation of the surface the coating solution is applied by means of flood-, dip-, spray-, or spincoating. [3] Thereafter, the solvent is evaporated and the resulting gel layer is thermally cured.

Since such coatings are normally well anchored on a surface, sol-gel coatings are furthermore well suited to act as primers between metals and organic polymers. ${ }^{[4]}$ Another application is the protection of scratch-sensitive metal surfaces (e.g. gold). By means of a thin anti-scratch coating the abrasion resistance is greatly improved without affecting the optical appearance of the surface. Furthermore, a welcome side effect is the increase of the heat-tinting temperature. ${ }^{[5]}$ Nevertheless, one of the important applications of sol-gel coatings is the fabrication of anti-adhesive layers. ${ }^{[6,7]}$ Such layers have numerous applications, especially in household or medical technology as they allow for less cleaning and improved hygiene. Such coatings further facilitate the removal of unwanted smearing such as graffiti. Several applications of sol-gel coatings are described in the following.

\section{Sol-Gel Coatings Containing Photoresponsive Azobenzene Moieties}

Surfaces displaying photo-switchable effects are of great interest for optical and optoelectronic applications, such as optical information storage or photoresponsive coatings. In this respect, very promising are sol-gel coatings containing azobenzene moieties, because they undergo reversible changes in surface wettability upon light exposure. Azobenzene isomerizes from the trans- to the cis configuration by irradiation with UV light with a wavelength of about $360 \mathrm{~nm}$. The reverse isomerization process occurs photochemically with visible light or thermally, since trans-azobenzene is thermodynamically more stable than cisazobenzene by $56 \mathrm{~kJ} / \mathrm{mol}$. The dipole moment of the cis isomer is considerably larger than that of the trans isomer. Therefore, the wetting properties of a coating containing azobenzene depend on the configuration of the azobenzene, whereby the cis azoben- 


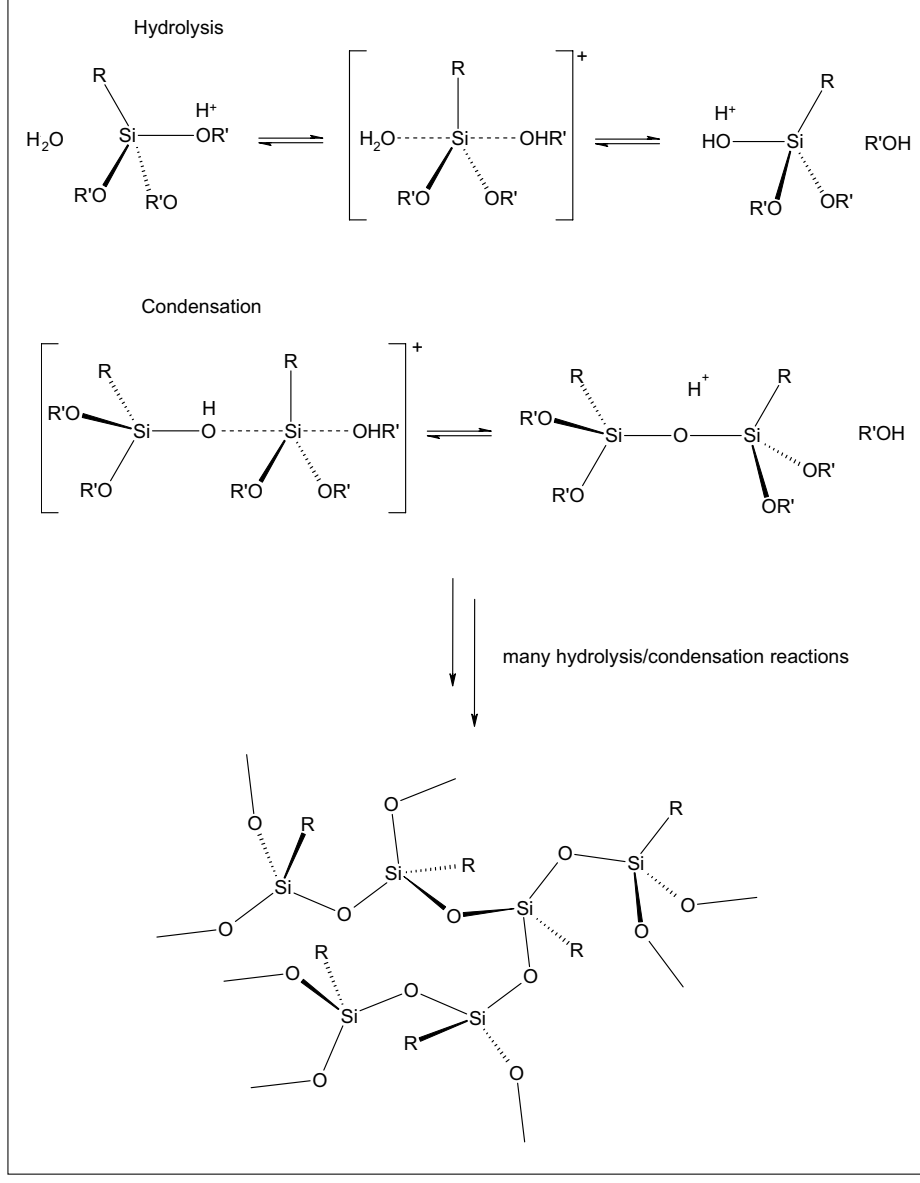

Scheme 1. Hydrolysis and condensation reactions for alkoxysilanes with acid catalysis

zene is more hydrophilic due to the larger dipole moment. A surface coated with azobenzene thus becomes more hydrophilic when irradiated with UV light. This effect was observed previously on self-assembled monolayers, ${ }^{[8,9]}$ but we describe more versatile sol-gel coatings on glass with silanes containing azobenzene and their wetting characteristics.

Synthesis of the azobenzene-silanes is shown in Scheme 2. The allylether 1 was prepared according to literature procedures. ${ }^{[10]}$ Hydrosilylation of $\mathbf{1}$ with trimethoxysilane and a platinum (II) catalyst resulted in the azobenzene-silane 2 . Hydrolysis and condensation of $\mathbf{2}$ with concentrated hydrochloric acid in isopropanol/hexane yielded a sol, which was applied to glass substrates. After drying and cross linking, water contact angles were measured. The coated glass slides were exposed to $360 \mathrm{~nm} \mathrm{UV} \mathrm{radia-}$ tion, and the contact angle was measured again. The contact angle decreased from 98 $\pm 5^{\circ}$ prior the UV treatment to $86 \pm 1^{\circ}$ after the treatment, leading to a more hydrophilic surface. The contact angle decreased due to the isomerization of the azobenzene towards the cis state and the corresponding increase in dipole moment. Thereafter, the glass slide was exposed to ambient light for $20 \mathrm{~h}$. The contact angle increased to $90 \pm 2^{\circ}$ making the surface more hydrophobic. The increase of water contact angle is due to the isomerization back to the trans state. This process could be repeated several times.

This example illustrates that one can design photosensitive coatings whose hydrophilicity can be modified by irradiation with UV light. Although the effect is small, it could probably be enhanced on a structured surface. Substantially larger photoswitched wettability on inverse opal coated with a self-assembled azobenzene monolayer has been reported.[11] These authors find that on such a rough surface, the contact angle of water changes from initially $141 \pm 3^{\circ}$ to $122 \pm 4^{\circ}$ after UV irradiation. The advantage of the present sol-gel coatings over self-assembled monolayers is that they can be applied to many different substrates and they offer interesting mechanical properties in addition.

\section{Antifreeze Coatings for Rotor Blades of Wind Turbines}

The sun is our largest energy source. It radiates approximately $10^{18} \mathrm{kWh}$ per year to the earth. This amount of energy corresponds to almost 10'000-fold of the present energy demand of humanity. About $2 \%$ of
$\stackrel{\mathrm{Br} \Upsilon}{\longrightarrow}$<smiles>C=CCOc1ccc(/N=N/c2ccccc2)cc1</smiles><smiles>CO[SiH2]CCCOc1ccc(N=Nc2ccccc2)cc1</smiles>

2

the energy of the solar radiation is transformed into wind. Theoretically, wind energy could therefore more than satisfy world's energy needs. The consequence of this large potential is only one of various factors making wind power the strongest expanding energy industry. Globally, installed wind power has more than tripled in the period from 2001 to 2005 . In order to fully utilize the existing wind potential in Europe, wind turbines have to be erected at sites that are endangered to icing. Particularly in Switzerland, the wind map shows that the winds are strongest at high altitudes. Favourable locations for wind turbines in Switzerland lie almost exclusively more than $800 \mathrm{~m}$ above sea level in a cold and turbulent climate, where icing is frequent. Yet, icing is fatal for wind turbines, as ice accumulation at the leading edge of the rotor blades alters their profile and finally impedes energy production altogether. Moreover, falling ice from iced rotor blades endangers passersby. Therefore, technical solutions are required that permit the operation of wind turbines under icing conditions. While ice accumulation represents an urgent problem for wind turbines, one faces similar difficulties with boats, airplanes, or power lines. To date, no convincing solutions are available.

An elegant solution for the prevention of ice accumulation would be an ice repellent coating. ${ }^{[12]}$ However, the development of such icephobic coatings represents a challenge, because ice sticks to everything. While several coatings and paints that should minimize ice accumulation are commercially available, it is known that that none of these products can avoid ice build-up. In fact, ice grows on those surfaces with the same speed as on other materials. Nevertheless, the adhesion force of ice is reduced, such that the ice can be removed more easily. Such icephobic coatings are normally selected according to their hydrophobicity, that is, their ability to repel water. A common working hypothesis to select such coatings is that a surface that has 
little affinity to water would also display weak ice adhesion. For example, Teflon is an example of such icephobic coating. ${ }^{[13]} \mathrm{A}$ Teflon surface is water repelling and shows equally a weak adhesion to ice. However, the mentioned working hypothesis does not always apply, and there is significant lack of knowledge concerning their anti-adhesive properties. In our opinion, no perfect icephobic coating exists.

Our approach to develop antifreeze coatings is based on the fifty-years-old observations of the Canadian scientist Scholander. He wondered how arctic fish could survive in waters that are colder than the freezing point of their blood, and suggested that there was 'antifreeze' in their blood. In the late 1960s the biologist DeVries isolated antifreeze proteins from the blood of Antarctic fish. ${ }^{[14]}$ Antifreeze proteins create a difference in the melting- and freezing temperature of water. The blood of the arctic fish freezes at $-2{ }^{\circ} \mathrm{C}$, whereas the ice crystals melt at about $0{ }^{\circ} \mathrm{C}$. This phenomenon is referred to as thermal hysteresis. It is believed that antifreeze proteins hinder the ice crystal growth by an adsorption-inhibition mechanism. They adsorb irreversibly to the ice surface and inhibit the growth of the ice crystals, which would be lethal to the fish. Recently, insects and beetles were reported to withstand even lower temperatures without freezing. In this case, one refers to hyperactive antifreeze proteins.

Our own as well as the research of others ${ }^{[15]}$ has shown that synthetic polymers show similar thermal hysteresis effects. Polyvinylalcohol is an example for such an 'antifreeze polymer' and our goal is to use such polymer on surfaces in order to obtain antifreeze coatings. In general, little is known about the behaviour of antifreeze proteins and antifreeze polymers on surfaces. If a supercooled water droplet hits a surface coated with an antifreeze polymer, it should not freeze, at least down to a certain minimal temperature, which is given by the activity of the coating. Even when the droplet freezes, a layer of liquid water between the ice and the coating will remain, and the ice can easily slip from the surface. The coating acts somewhat similar to a heating device. Since a layer of liquid water remains on the surface, the ice cannot bind to the surface. Fig. 1 shows a micrograph of such an experiment. To the right, ice crystals on the uncoated glass can be seen, whereas to the left liquid water droplets at the coated section can be observed. Since the entire slide is at the same temperature, this experiment clearly illustrates that such a coating may indeed prevent freezing of water on surfaces. We are currently investigating how other polymers on glass substrates prevent ice formation, and we hope that this approach will lead towards useful coatings to protect rotor blades of wind turbines from icing.

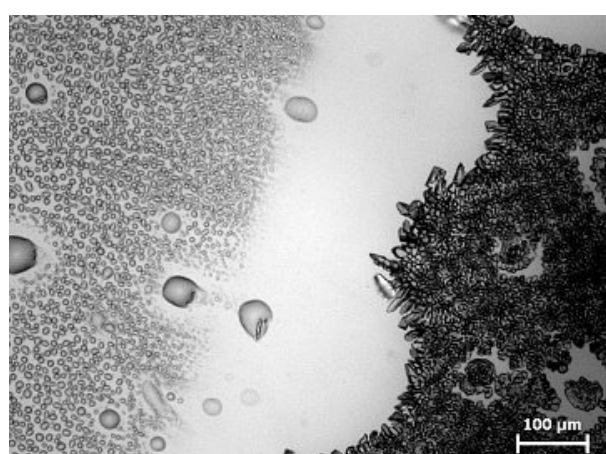

Fig. 1. An antifreeze coating. On the left: liquid water on coated glass. On the right: ice on uncoated glass. Both sides have the same temperature $\left(-18^{\circ} \mathrm{C}\right)$.

\section{Coatings with Lime-repellent Properties}

Calcification, or scaling, is a problem known to everyone. Thereby, sparingly soluble calcium carbonate precipitates and adheres to surfaces, forming crusts of lime that are difficult to remove. The formation of calcium carbonate deposits on surfaces is most important in hard waters. Water hardness is defined as the concentration of dissolved alkaline earth ions, principally calcium and to a lesser extent, magnesium Hard water further increases the amount of detergents needed for cleaning, and further influences the taste and appearance of beverages. Hard water occurs in regions rich in limestone, ${ }^{[16]}$ and originates from the dissolution of lime, $\mathrm{CaCO}_{3}$, or dolomite, $\mathrm{CaMg}\left(\mathrm{CO}_{3}\right)_{2} \cdot{ }^{[17]}$ During the dissolution process, carbonic acid reacts with the carbonate forming hydrogen carbonate according to the reaction:

$$
\begin{aligned}
& \mathrm{CaCO}_{3}+\mathrm{CO}_{2}+\mathrm{H}_{2} \mathrm{O} \rightleftarrows \\
& \mathrm{Ca}^{2+}+2 \mathrm{HCO}_{3}^{-}
\end{aligned}
$$

Note that calcium hydrogen carbonate $\mathrm{Ca}\left(\mathrm{HCO}_{3}\right)_{2}$ is well soluble in water, quite in contrast to the sparingly soluble $\mathrm{CaCO}_{3} \cdot{ }^{[18]}$ Equally, acid rain and nitrification can lead to dissolution of lime. The dissolution and precipitation of lime are therefore complex chemical processes in which $\mathrm{pH}$-dependent equilibria are involved. Calcification is the inverse of the process given in reaction (1).

Scaling can be avoided by water softening, and the latter can be achieved by ion exchange. Thereby, calcium and magnesium ions are replaced by sodium ions. This approach is used in dish washers, for example, in order to spare the heating elements and to avoid stains on dishes. ${ }^{[19]}$ Complete demineralization can be achieved by a combination of cation- and anion exchangers. Another method to avoid scaling is by adding complexing agents, which react with the calcium. Polyphosphates were often employed, but they lead to over-fertilization of waters and have therefore been abandoned.
Modern detergents use zeolites as additives, which act as cation exchangers.

A different approach to avoid scaling is to inhibit the adhesion of lime at surfaces. Since little is known about how lime binds to a material, we have been investigating various substrates with respect to their ability to repel calcification. Plastics, metals, and glass were covered by an aqueous solution of $\mathrm{CaCl}_{2} / \mathrm{NaHCO}_{3}$ from which $\mathrm{CaCO}_{3}$ slowly precipitated. After calcification, the samples were dipped in water to remove weakly adhering $\mathrm{CaCO}_{3}$ and dried. The resulting surface coatings were investigated by light microscopy and scanning electron microscopy. After $3 \mathrm{~h}$ calcification three calcium carbonate modifications have been observed, namely vaterite, aragonite, and calcite. ${ }^{[20]}$ After $16 \mathrm{~h}$ the vaterite modification disappeared in favour of aragonite and calcite, which are the thermodynamically more stable modifications. After $64 \mathrm{~h}$, calcite was the predominant modification on all surfaces. The hydrophobic polymers, such as poly(ethylene) and poly(vinylidene fluoride), calcified less after $3 \mathrm{~h}$, however, after $16 \mathrm{~h}$ the differences between the individual materials became smaller and after $64 \mathrm{~h}$ all surfaces were homogeneously calcified. As hydrophobic surfaces are anticipated to display only weak interactions with the ionic calcium carbonate, we investigated highly hydrophobic surfaces, among them superhydrophobic and easy-to-clean coatings. Still, after $64 \mathrm{~h}$ calcification, those surfaces were calcified just like all others. One concludes that hydrophobicity therefore does not hinder calcification.

It appeared to us that a more promising approach could be the development of hydrophilic and water-soluble polymer coatings. A promising candidate to obtain hydrophilic coatings is poly(ethylene glycol) (PEG). This water-soluble polymer, also referred to as polyethylene oxide (PEO), is obtained by polymerization of ethylene oxide and has the repeating subunit $\mathrm{CH}_{2} \mathrm{CH}_{2} \mathrm{O}-$. Depending on their molecular weights, PEGs are liquids or low-melting solids. PEGs are soluble in water, methanol, benzene and dichloromethane, but are insoluble in diethyl ether and hexane. PEGs can be coupled to aliphatic chains to produce non-ionic surfactants. PEG has a low toxicity and is used in a variety of products. All those properties make PEG an interesting candidate for the preparation of hydrophilic and liquid-like surfaces.

A methylated PEG with an average molecular weight of $350 \mathrm{~g} / \mathrm{mol}$ is bound via a propyl-spacer to a trimethoxysilane moiety to a glass surface. After usual sol-gel reactions, a coating of PEG-silane on glass can be realized. Such coatings efficiently inhibit calcification (see Fig. 2). If plastic substrates are pre-treated by low pressure oxygen plasma, they can also be coated by 


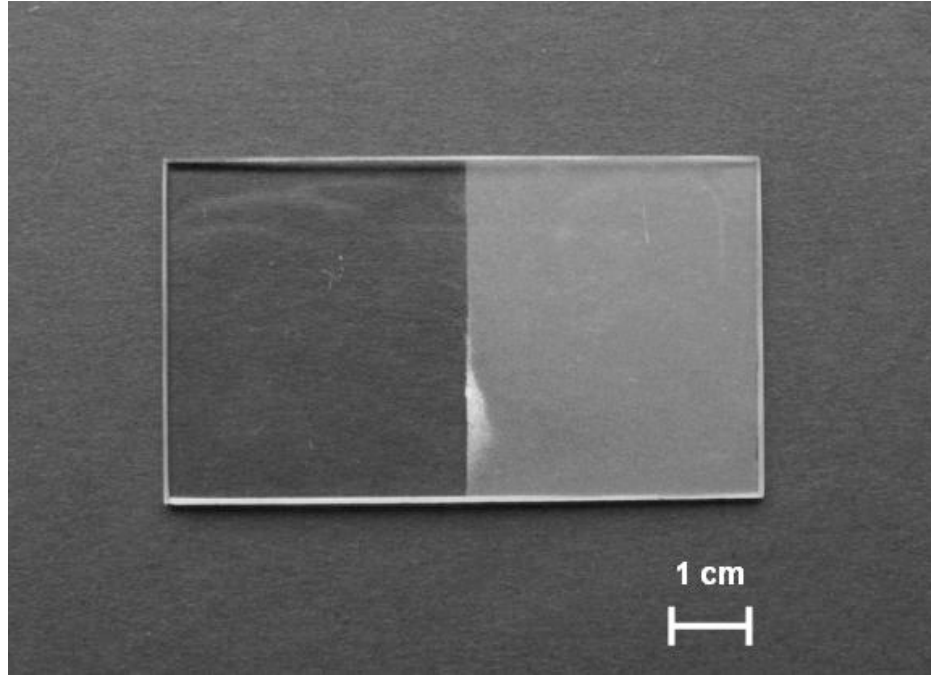

Fig. 2. Calcification of a coated glass substrate. The left side is coated and therefore not calcified, whereas the right side is not coated and therefore covered with lime.

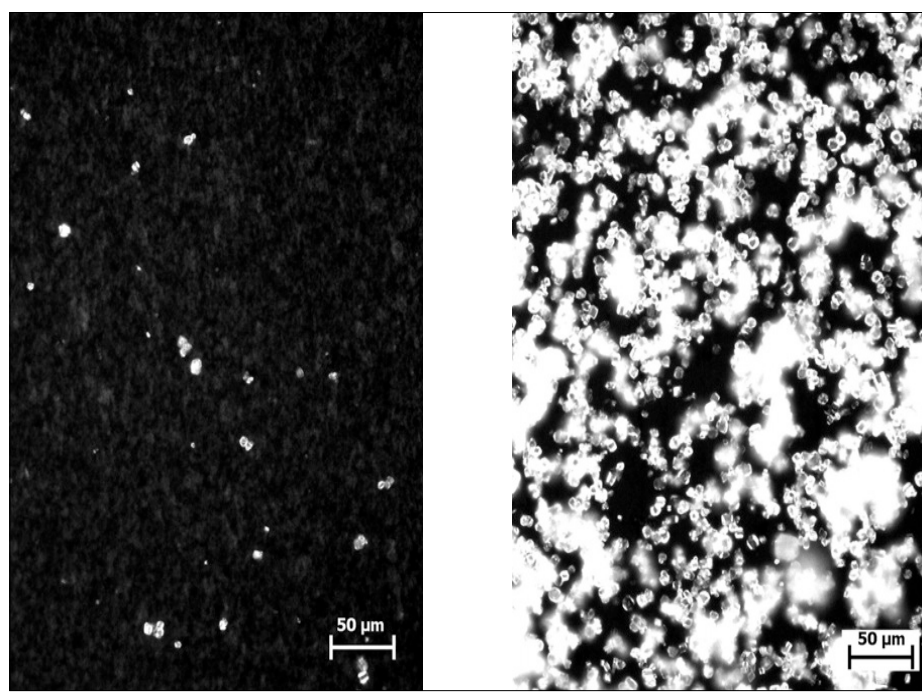

Fig. 3. Microscopic picture of a calcified poly(ethylene) substrate. The left side is coated and the right side is uncoated.
PEG. Fig. 3 shows a microscopic picture of a calcified poly(ethylene) substrate. On the left, the substrate coated with PEG is shown, and in this area only a few calcium carbonate crystals are observed. On the right, on the uncoated side, large deposits of calcium carbonate crystals are observed. At present, we are investigating the longterm stability of such coatings.

\section{Aerogels as Thermal Insulators in Construction}

Aerogels are solid sol-gel materials with an extremely low density. They are commonly produced from silica sols by replacing the solvent with gas, resulting in brittle silica foams. Aerogels possess the lowest thermal conductivity of all solid materials.[21] Their flameproof properties represent another advantage. For these reasons, they belong to the best heat-insulating materials available. The low thermal conductivity is a consequence of the porous structure of aerogels, leading to high porosities (up to 95\%) and substantial inner surface areas. ${ }^{[22]}$ Thereby, the porosity is defined as the volume of the pores divided by the total volume of the aerogel. These features of aerogels represent major advantages as insulating materials in construction. For these applications, however, aerogels have also some disadvantages, particularly, their high price due to the complex manufacturing process and their poor mechanical stability.

Silicon dioxide aerogel is produced by supercritically drying a hydrogel consisting of colloidal silica nanoparticles. ${ }^{[23]}$ The process starts with a silica precursor, such as tetraethoxysilane, mixed with ethanol, water and an acid catalyst to form a silicon dioxide gel. Ethanol is removed by supercritical drying. During this process, ethanol is first replaced by acetone and then by liquid carbon dioxide. The carbon dioxide is brought above its critical point and is slowly removed. This complicated procedure is necessary to avoid gas-liquid phase separation during the drying process in order to conserve the fragile silica network.

In order to produce aerogels in large quantities and to make them commercially interesting, it is necessary to avoid the costly supercritical drying process. Therefore, there is a substantial interest to develop alternative procedures to allow an exchange of liquids against air from the pore space. However, drying silica gels to obtain aerogels under atmospheric conditions is difficult, as capillary forces induce shrinking of the gel. Thereby, the hydroxy groups on the pore surfaces react and form new siloxane bridges. With progressive drying, the mesh becomes stiffer, leading to gel shrinkage and rupture. ${ }^{22]}$

In 1992 Smith et al. described the first synthesis of aerogels with drying under conventional conditions. ${ }^{[24]}$ Their procedure is based on a chemical modification of the inner surface of the gel. For example, one uses chlorotrimethylsilane, which reacts with the hydroxy surface groups, leading to an unreactive and hydrophobic inner surface. Upon drying, the gel shrinks as usual, but because the inner surface is unreactive, the shrinkage is reversible. After drying, the gel expands almost to its original size, which is referred to as the 'springback effect'. [22] The production of aerogels by such 'undercritical drying' is an active topic of research, as the process is potentially cheaper and makes aerogels produced in this fashion economically interesting. However, given the poor stability of aerogels they have to be reinforced, for example, by incorporating fibres. As the re- action of the gel with chlorotrimethylsilane liberates large amounts of hydrochloric acid, one can only use fibres that are stable in acidic media. Unfortunately, many fibres used are not stable against acid.

We fabricate high-porosity aerogels using water glass and tetraethylorthosilicate (TEOS) as precursors. In the case of water glass, the sodium ions were first exchanged against protons. The precursors were dissolved in water at different concentrations. After treating the aerogel with chlorotrimethylsilane, and subsequent drying, the porosity was determined.

Fig. 4 shows the porosity of the aerogel as a function of the mass fraction of $\mathrm{SiO}_{2}$. One can see that at low mass fractions $(<8 \%)$ TEOS leads to higher porosities than water glass. At higher mass fractions $(>8 \%)$, the aerogel from water glass has a higher porosity. TEOS was thus chosen as the appropriate precursor, since it leads to higher porosities.

As mentioned above, the treatment with chlorotrimethylsilane forms hydrochloric acid, which is detrimental to the reinforcing fibres. We therefore investigated the use of trimethylsilyl acetate as an alternative reagent, as it forms weakly acidic acetic acid upon reacting with the gel. Fig. 5 shows the porosity of the aerogel as a function of the amount of trimethylsilyl acetate employed. It can be seen that about $6 \mathrm{~g}$ trimethylsilyl acetate per gram gel are needed to obtain a maximal porosity of about $90 \%$. Below this concentration, the inner surface of the aerogel is not completely passivated and the springback-effect is therefore reduced.

\section{Conclusion}

The present article discusses various applications of sol-gel processes to obtain 


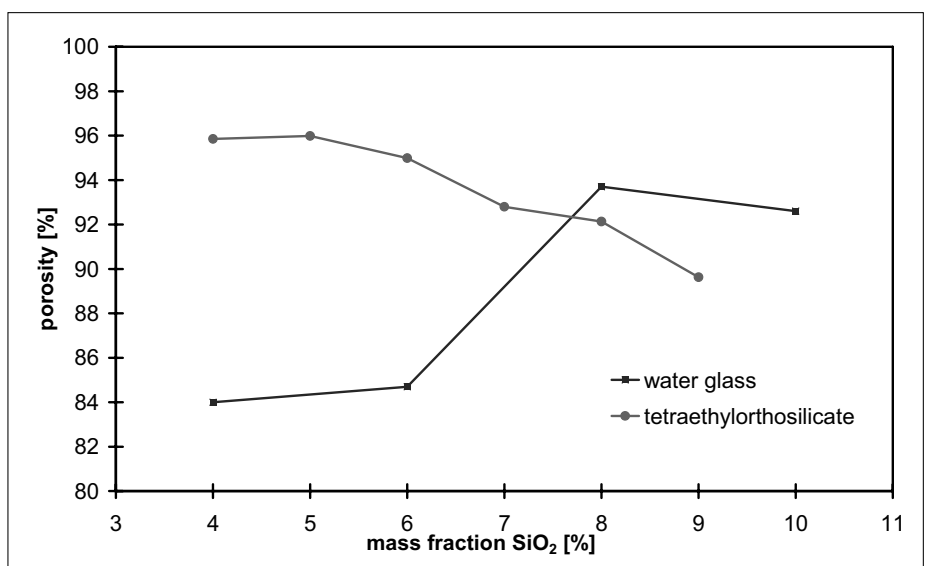

Fig. 4. Porosity of the aerogel as a function of $\mathrm{SiO}_{2}$ content for water glass and tetraethylorthosilicate

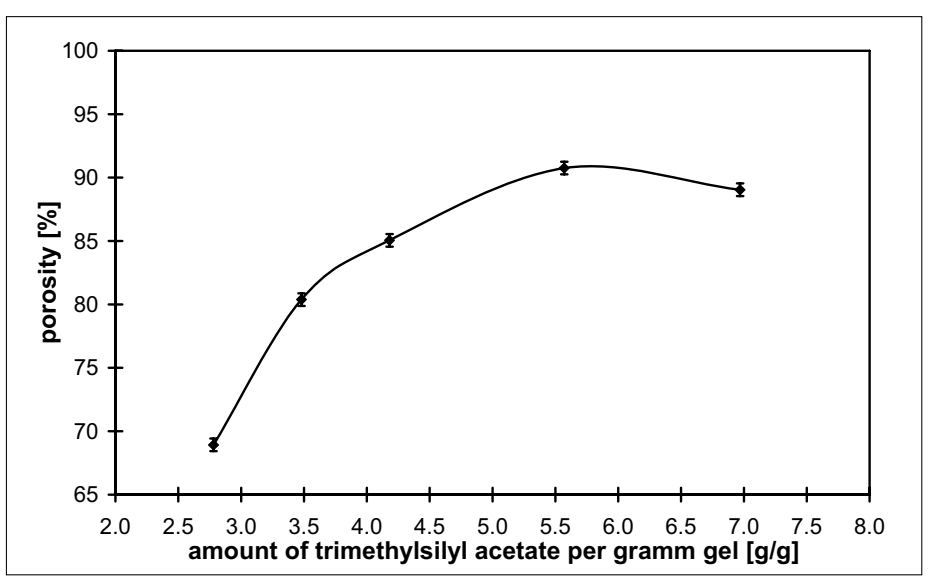

Fig. 5. Porosity of the aerogel as a function of trimethylsilyl acetate used coatings and aerogels. Such processes are based on hydrolysis and condensation reactions of alkoxysilanes leading to a siloxane nanocondensate. Such condensates show excellent adhesion properties and are applicable to a wide variety of surfaces. The technique is illustrated with sol-gel coatings having various properties. Photosensitive coatings are obtained by silane coupling of azobenzene moieties, while lime repellent or ice repellent coatings are obtained by coupling various polymers. The preparation of aerogels can be improved by selecting specific silanes for the 'coating' of the inner surface. The list is not exhaustive, and due to the versatility of the technique we expect that many more applications will emerge in the near future.

\section{Acknowledgements}

We wish to thank N. Weiler, G. Meola, L. Meier, F. Zuber, T. Glättli, S. Brunner and B. Vetterli for their contributions. Financial support from KTI/CTI and BFE is gratefully acknowledged. We thank Geberit International $\mathrm{AG}$ and Clariant for financial support.

Received: May 23, 2008
[1] L. L. Hench, J. K. West, Chem. Rev. 1990, 90, 33.

[2] H. Bötcher, Materialwiss. Werkstofftech. 2001, 32, 759 .

[3] T.Brock, M.Groteklaes, P.Mischke, in 'Lehrbuch der Lacktechnologie', Ed. U. Zorll, C. R. Vincentz, Hannover, 1998, p. 284.

[4] U. Deschler, P. Kleinschmidt, P. Panster, Angew. Chem. 1986, 98, 237.

[5] F. Auer, C. Roth, J. Harenburg, DE Patent Publication No. DE 19957325 A1 2001.

[6] H. J. Jeong, D. K. Kim, S. B. Lee, S. H. Kwon, K. Kadono, J. Colloid Interface Sci. 2001, 235, 130.

[7] B. Mahltig, H. Böttcher, J. Sol-Gel Sci. Technol. 2003, 27, 43.

[8] F. Hamelmann, U. Heinzmann, U. Siemeling, F. Bretthauer, J. Vor der Brüggen, Appl. Surf. Sci. 2004, 222, 1.

[9] L. M. Siewierski, W. J. Brittain, S. Petrash, M. D. Foster, Langmuir 1996, 12, 5838.

[10] L. Claisen, O. Eisleb, Justus Liebigs Ann. Chem. 1913, 401, 44.

[11] H. Ge, G. Wang, Y. He, X. Wang, Y. Song, L. Jiang, D. Zhu, ChemPhysChem 2006, 7, 575 .

[12] C. Laforte, J. L. Laforte, J. C. Carrière, The Tenth International Workshop on Atmospheric Icing of Structures, IWAIS, 2002.
[13] S. Frankenstein, A. M. Tuthill, J. Cold Reg. Engrg. 2002, 16, 83.

[14] A. L. De Vries, D. E. Wohlschlag, Science 1969, $163,1074$.

[15] T. Inada, S. S. Lu, Chem. Phys. Lett. 2004, $394,361$.

[16] W. Kölle, 'Wasseranalysen - richtig beurteilt. 2. Auflage', Wiley-VCH, 2003.

[17] T. O. West, A. C. McBride, Agricult. Ecosys. Environ. 2005, 108, 145.

[18] J. C. Jamieson, J. Chem. Phys. 1953, 21, 1385.

[19] M. A. Dia, S. M. Lo, M. Pontie, H. Bagan, C. K. Diawara, M. Rurneau, Comptes Rendus Chimie 2006, 9, 1260.

[20] L. N. Plummer, E. Busenberg, Geochim. Cosmochim. Acta 1982, 46, 1011.

[21] F. Schwertfeger (Hoechst AG), Future Special Science - das Hoechst Magazin 1997, 2, 28.

[22] N. Hüsing, U. Schubert, Angew. Chem. 1998, 110, 22

[23] P. H. Tewari, A. J. Hunt, K. D. Lofftus Mater. Lett. 1985, 3, 363.

[24] D. M. Smith, R. Desphande, C. J. Brinker in 'Better Ceramics Through Chemistry', Mater. Res. Soc. Symp. Proc. 1992, p. 567. 